\title{
Magnetic resonance for assessment of disease activity and severity in ileocolonic Crohn's disease
}

\author{
J Rimola, ${ }^{1}$ S Rodriguez, 0 García-Bosch, ${ }^{2}$ I Ordás, ${ }^{2}$ E Ayala, ${ }^{3}$ M Aceituno, ${ }^{2}$ M Pellisé, ${ }^{2}$ \\ C Ayuso, ${ }^{1}$ E Ricart, ${ }^{2}$ L Donoso, ${ }^{1}$ J Panés ${ }^{2}$
}

${ }^{1}$ Department of Radiology, Hospital Clínic de Barcelona, IDIBAPS, Centro de

Investigación Biomédica en Red de Enfermedades Hepáticas y Digestivas (CIBEREHD), Barcelona, Spain; ${ }^{2}$ Department of Gastroenterology, Hospital Clínic de Barcelona, IDIBAPS, Centro de Investigación Biomédica en Red de Enfermedades Hepáticas y Digestivas (CIBEREHD), Barcelona, Spain; ${ }^{3}$ Department of Epidemiology, Hospital Clínic de Barcelona, IDIBAPS, Centro de Investigación Biomédica en Red de Enfermedades Hepáticas y Digestivas (CIBEREHD),

Barcelona, Spain

Correspondence to: Dr J Panés, Department of Gastroenterology, Hospital Clínic of Barcelona, Villarroel 170, 08036 Barcelona, Spain; jpanes@clinic.ub.es

Revised 20 November 2008 Accepted 11 December 2008 Published Online First

6 January 2009

\begin{abstract}
Objective: Assessment of disease extension and activity is crucial to guide treatment in Crohn's disease. The objective of the current cross-sectional study was to determine the accuracy of MR for this assessment. Design: 50 patients with clinically active $(n=35)$ or inactive $(n=15)$ Crohn's disease underwent ileocolonoscopy (reference standard) and MR. T2-weighted and precontrast and postcontrast-enhanced T1-weighted sequences were acquired. Endoscopic activity was evaluated by CDEIS (Crohn's Disease Endoscopic Index of Severity); in addition endoscopic lesions were classified as absent, mild (inflammation without ulcers) or severe (presence of ulceration).
\end{abstract}

Results: The comparison of intestinal segments with absent, mild and severe inflammation demonstrated a progressive and significant $(p<0.001)$ increase in the following MR parameters: wall thickness, postcontrast wall signal intensity, relative contrast enhancement, presence of oedema, ulcers, pseudopolyps and lymph node enlargement. Independent predictors for CDEIS in a segment were wall thickness $(p=0.007)$, relative contrast enhancement $(p=0.01)$, presence of oedema $(p=0.02)$ and presence of ulcers at MR $(p=0.003)$. There was a significant correlation $(r=0.82, p<0.001)$ between the CDEIS of the segment and the MR index calculated according to the logistic regression analysis coefficients. The MR index had a high accuracy for the detection of disease activity (area under the receiver operating characteristic (ROC) curve 0.891 , sensitivity 0.81 , specificity 0.89 ) and for the detection of ulcerative lesions (area under the ROC curve 0.978 , sensitivity 0.95 , specificity 0.91 ) in the colon and terminal ileum.

Conclusion: The accuracy of MR for detecting disease activity and assessing severity brings about the possibility of using MR as an alternative to endoscopy in the evaluation of ileocolonic Crohn's disease.

Assessment of disease extension and activity is crucial in patients with Crohn's disease (CD) to determine the therapeutic strategy, and has important prognostic implications. This assessment usually relies on endoscopy. ${ }^{1}$ However, endoscopy cannot always be complete, and there are several drawbacks related to the invasiveness, procedurerelated discomfort, risk of bowel perforation and relatively poor patient acceptance. This fact has led to the search for alternative techniques to assess disease extension and severity in inflammatory bowel disease (IBD) including sonography and $\mathrm{CT} .{ }^{2}$ Exposure to ionising radiation is a relevant limitation of the latter, especially in light of the patients' young age. ${ }^{3}$ We and others have proposed the use of sonography for evaluation of IBD activity as an alternative to endoscopy, but assessment of the intestine by this technique is frequently incomplete due to gas interposition. ${ }^{4}$

Magnetic resonance (MR) has the potential to overcome these limitations. It is characterised by a very high soft tissue contrast, lack of ionising radiation and lower incidence of adverse events related to the intravenous contrast employed compared with CT. Although several MR characteristics of the bowel have been described in patients with $C D$, no studies have specifically addressed the relative usefulness of each of these $M R$ signs in the assessment of inflammatory activity and severity, and most protocols relied only on measurement of wall thickness and degree of enhancement after administration of paramagnetic contrast. Using the latter parameters, some previous reports ${ }^{5-9}$ produced controversial results, and methodological differences make comparisons difficult. Despite its potential usefulness, a major drawback to the routine use of $M R$ in the assessment of $\mathrm{CD}$ is the lack of precise criteria to define bowel involvement by $\mathrm{CD}$, and quantify the severity of inflammatory lesions.

Therefore, the aims of this cross-sectional study were to characterise the MR patterns of the colon and the terminal ileum in patients with active and inactive $\mathrm{CD}$; to establish the MR changes indicative of mild and severe intestinal lesions; and to provide a quantitative index of disease activity based on MR findings.

\section{PATIENTS AND METHODS}

\section{Patients and examinations}

Between June 2007 and April 2008, 50 patients with an established diagnosis of $\mathrm{CD}$ of at least 6 months duration, with or without clinical symptoms of activity, were prospectively studied at a single centre. During the same period, two patients refused to participate in the study because of personal reasons: one patient preferred to avoid MR exploration due to claustrophobia, and in another patient MR examination could not be completed due to intolerance of the enema. Patients underwent complete clinical assessment, had blood samples obtained for cell blood counts and C-reactive protein (CRP) measurements, and underwent conventional ileocolonoscopy and MR examination within 2 days. Clinical disease activity was assessed using the Harvey-Bradshaw score. ${ }^{10}$ Investigators performing ileocolonoscopy or $\mathrm{MR}$ were unaware of clinical data or of the results of other examinations. All patients gave their informed consent to participate in the study after 
approval of the project by the ethics committee of the Hospital Clínic of Barcelona.

\section{Endoscopic data collection}

Ileocolonoscopy was considered the reference standard for the evaluation of IBD extension and severity. All patients underwent endoscopy 4-24 $\mathrm{h}$ before or after the MR examination. Patients followed a bowel cleansing protocol with oral ingestion of $3000-4000 \mathrm{ml}$ of an iso-osmotic polyethylene glycol (PEG) and electrolyte solution (Bohm laboratories, Fuenlabrada, Spain) on the evening before examination. Endoscopies were performed under anaesthesia with propofol (Mayne Pharma, Madrid, Spain) and remifetanyl (GlaxoSmithKline, Madrid, Spain). The severity and extent of inflammatory lesions were evaluated using the Crohn's Disease Endoscopic Index of Severity (CDEIS). ${ }^{1}$ For CDEIS calculation, the endoscopic variables were as originally defined: deep ulcers and superficial ulcers (presence or absence), ulcerated surface and affected surface (evaluated on a $10 \mathrm{~cm}$ linear analogue scale), and ulcerated and non-ulcerated stenosis. These variables were evaluated in the terminal ileum, ascending colon, transverse colon, descending and sigmoid colon, and the rectum.

For comparison of endoscopic and MR findings in each segment, a calculation of endoscopic activity of the segment was performed by dividing the colon into five segments instead of four as for the conventional CDEIS calculation (ascending colon, transverse colon, descending colon, sigmoid colon and rectum) and scoring the CDEIS variables in each of these segments and the terminal ileum. The score of ulcerated or nonulcerated stenosis was imputed to the affected segment. In addition, a classification of lesion severity of each segment was performed by considering three categories: (1) absence of lesions; (2) presence of inflammatory lesions without ulceration, including erythema, oedema, pseudopolyps and aphtae; and (3) presence of superficial or deep ulcerations.

The endoscopist completed an endoscopic scoring sheet immediately after colonoscopy. All procedures were performed by two gastroenterologists with $>10$ years experience in conventional colonoscopy using standard equipment (CFO 140 L; Olympus, Japan), and after a period of joint training for CDEIS scoring.

\section{MR acquisition}

In most cases MR and ileocolonoscopy were performed on the same day, under the bowel cleansing protocol described previously, without any additional preparation for MR. In the few instances that the two techniques were not performed on the same day bowel cleansing with 1000-2000 $\mathrm{ml}$ of PEG solution was given $4 \mathrm{~h}$ before performing MR.

All MR examinations were performed using a 3.0 T MR unit (TrioTim; Siemens Medical Solutions, Erlangen, Germany). Patients were placed in the supine position in the MR imager. This position is more comfortable for patients. A combination of two surface coils was used for signal reception to allow coverage of the whole abdominal area. To reduce bowel peristalsis, $40 \mathrm{mg}$ of hyoscine butylbromide (Buscapina; Boehringer Ingelheim, Sant Cugat, Spain) were injected intravenously.

To achieve an adequate distension of the distal ileum, $45 \mathrm{~min}$ before the MR each patient was required to drink $1500 \mathrm{ml}$ of iso-osmotic PEG and electrolyte solution. Saline was retrogradly instilled into the colon through a flexible rectal balloon catheter (Silkolater Teleflex Medical, Alicante, Spain). The volume of warm water was adjusted for each patient to avoid abdominal pain or discomfort, ranging from 1000 to $2000 \mathrm{ml}$. The procedure was well tolerated.

Initially a true fast imaging with a steady precession sequence was acquired in the coronal plane to ensure optimal colon distension. Then, the acquisition protocol outlined in table 1 was performed. VIBE (volumetric interpolated breath-hold examination) sequences were acquired before and $70 \mathrm{~s}$ after intravenous administration of $0.2 \mathrm{ml} / \mathrm{kg}$ body weight of gadolinium chelate (gadodiamide $0.5 \mathrm{mmol} / 1$ OminscanAmersham, Madrid, Spain) at a rate of $2 \mathrm{ml} / \mathrm{s}$.

\section{MR image analysis}

To establish disease extension by $M R$, we used the same division into six segments as was used in colonoscopy (distal ileum, ascending, transverse, descending, sigmoid colon and rectum). Image analysis was performed using a dedicated postprocessing workstation (Leonardo; Siemens AG Medical Solutions). The following were studied by MR in each colonic segment and in the terminal ileum: bowel wall thickness $(\mathrm{mm})$, presence of mucosal ulceration (defined as deep depressions in the mucosal surface), presence of mural oedema (hyperintesity on T2-wedged sequences of the colon wall relative to the signal of the psoas muscle), presence of pseudopolyps in the lumen, enlarged $(>1 \mathrm{~cm})$ regional mesenteric lymph nodes, quantitative measurement of wall signal intensity (WSI) before and after intravenous contrast administration measured in VIBE sequences, and relative contrast enhancement (RCE) of the intestinal wall. Quantitative measurements of WSI were obtained from the areas with the greatest thickening. WSI corresponds to the average of three WSI measurements. RCE was calculated according the following formula: $\mathrm{RCE}=((\mathrm{WSI}$ postgadolinium-WSI pregadolinium)/(WSI pregadolinium) $) \times 100 \times($ SD noise pregadolinium/SD noise postgadolinium), where SD noise pregadolinium corresponds to the average of three SDs of the signal intensity measured outside of the body before gadolinium injection, and SD noise postgadolinium corresponds to the SD of the same noise after gadolinium administration. ${ }^{11}$

\section{Statistical analysis}

Differences in qualitative MR findings were tested with the $\chi^{2}$ test; differences in quantitative measures were tested using analysis of variance (ANOVA) followed by the Bonferroni posthoc test. Correlations between the CDEIS, HarveyBradshaw index, CRP and MR index scores were measured by the Spearman rank coefficient.

For defining the MR findings that were independent predictors of disease activity, a binary logistic regression was

Table 1 Protocol for MR image acquisition

\begin{tabular}{llllll}
\hline & Plane & $\begin{array}{l}\text { Slice } \\
\text { thickness } \\
(\mathbf{m m})\end{array}$ & FOV & TR/TE $(\mathbf{m s})$ & Flip angle \\
\hline $\begin{array}{l}\text { True-FISP } \\
\begin{array}{l}\text { T2-HASTE } \\
\text { with fat }\end{array}\end{array}$ & Coronal & 4 & $400 \times 400$ & $3.46 / 1.53$ & 50 \\
$\begin{array}{l}\text { saturation } \\
\text { T2-HASTE }\end{array}$ & 5 & $300 \times 300$ & $1000 / 99$ & 150 \\
VIBE & Coronal & 5 & & & \\
\hline
\end{tabular}

FISP, fast imaging with steady precession; FOV, field of view; HASTE, half-Fourier single-shot turbo spin-echo; TE, echo time; TR, repetition time; VIBE, volumetric interpolated breath-hold examination. 
used with the presence of an endoscopic lesion (of any type) as the dependent variable, and with the presence of oedema, ulcerations, pseudopolyps, wall thickness and RCE in MR as independent variables. For defining the independent predictors of the presence of ulcerations at endoscopy, a binary logistic regression was used, with this endoscopic finding as dependent variable, and the same $M R$ variables listed above as independent variables. The multivariate procedures were validated by bootstrap bagging with 1000 samples. Development of an MR index of disease activity was based on the findings of the individual segments. Since distribution of the values for CEDEIS was not normal, with $60 \%$ of segments having no significant lesions, a tobit regression model was applied. In that model, the calculated CDEIS per segment was used as the dependent variable, and again the presence of oedema, ulcerations, pseudopolyps, wall thickness and RCE in MR were introduced as independent variables. The accuracy of the scores determined by $M R$ for the prediction of the presence of active disease and the presence of severe lesions was assessed by calculating receiver operating characteristic (ROC) curves.

Interobserver agreement between paired evaluations of $M R$ by two radiologists (JR and SR) was performed through kappa statistics ${ }^{12}$ for comparisons of qualitative variables (presence of oedema, ulcers, pseudopolyps and lymph nodes), and through the intraclass correlation coefficient ${ }^{13}$ for assessing the reproducibility level of quantitative $M R$ measurements (wall thickness, basal signal intensity, contrast signal intensity and RCE).

\section{Statistical power}

We estimated that by studying 50 patients at least 200 colonic segments and 40 ileal segments would be available for examination (colonoscopy completed in $90 \%$ and ileoscopy in $80 \%$ ). Of those $50 \%$ would be normal, $25 \%$ would have nonulcerative lesions and $25 \%$ would have ulcerative lesions. This number provides the ability to detect differences of $20 \%$ for the presence of activity, and $25 \%$ for the presence of ulcerations among those with disease activity with an alpha risk of 0.05 and beta of 0.1 .

A $p$ value of $<0.05$ was considered significant. Calculations were done with the SPSS package version 15 (SPSS, 1989-2006, Chicago, Illinois, USA).

\section{RESULTS}

\section{Patients}

Clinical and biological characteristics of the 50 patients included in the study are provided in table 2. According to the HarveyBradshaw index, 15 patients were in clinical remission (index $\leqslant 4)$ and 35 patients had active disease (index $>4$ ). CRP was elevated relative to reference values (normal $\leqslant 8 \mathrm{mg} / \mathrm{l}$ ) in 35 patients. Complete endoscopic evaluation of the colon with intubation of the ileum through the ileocecal valve or an ileocolonic anastomosis was achieved in 36 patients (72\%). Reasons for incomplete ileocolonoscopy were disease severity $(n=3)$, stenosis $(n=3)$ or technical impossibility $(n=8)$. The total number of colonic segments examined, considering the extension of examinations and previous resection surgery, was 213. Of these, 130 were without lesions, 43 had mild lesions including erythema, oedema, pseudopolyps or aphtoid ulcers, and 40 segments had severe lesions with superficial and/or deep ulcers. Tolerance of MR examination was in general very good. The average duration of the entire MR examination within the
$M R$ imaging unit was $\sim 25$ min. Only three patients, all with severe rectal lesions, complained of tenesmus upon instillation of the enema that did not preclude completion of the examination.

\section{MR findings according to endoscopic lesion severity}

As shown in table 3 and fig 1 , the majority of MR findings had a close correlation with the severity of endoscopic lesions. Oedema on MR examination was present in $77.5 \%$ of colonic segments with ulcers at endoscopy, was significantly less frequent in segments with inflammatory lesions without ulcers $(20.9 \%)$ and absent in endoscopically normal segments. Ulcers were identified by MR in $65 \%$ of patients with ulcers at endoscopy, were very rare in segments with mild inflammation $(7.0 \%)$ and were never observed in endoscopically normal mucosa. The presence of enlarged lymph nodes and pseudopolyps was less prevalent, but also significantly more frequent in patients with ulcers compared with the other groups of patients (table 3). The kappa measure of agreement between endoscopy and MR for identification of ulcers was 0.71, and for pseudopolyps 0.86, although the sensitivity of $M R$ for the detection of segments with ulcerative lesions was markedly increased when not only direct identification of ulcers, but also wall thickening and REC were considered (see below).

Figure 1 depicts the changes in quantitative MR parameters according to endoscopic severity of colonic lesions. The increase in wall thickness paralleled the endoscopic severity of lesions, with a significant increase in segments with inflammatory lesions without ulcers compared with those with normal mucosa, and again significantly increased in segments with ulcers compared with non-ulcerative inflammatory lesions. In contrast, basal signal intensity was similar in all groups. After intravenous injection of contrast, WSI was markedly elevated in segments with ulceration relative to segments with inflammation without ulcers, and also in the latter compared with normal segments. RCE also paralleled the severity of endoscopic lesions with significant differences between segments with normal mucosa, segments with mild lesions and segments with ulcers.

MR changes were similar in all colonic segments, with the exception of lymph node enlargement that was predominantly associated with the presence of severe endoscopic lesions in terminal ileum, ascending colon and rectal regions. The characteristics and magnitude of MR changes in the ileum were similar to those of the colon, with significant $(p<0.05)$ increases in the proportion of patients having oedema, ulcers identified at $\mathrm{MR}$, and lymph nodes in the ileocecal region, as the severity of endoscopic lesions increased from normal to presence of any lesion, and from non-ulcerative lesions to presence of ulcers Pseudopolyps were not identified in the ileum. Also a significant increase in wall thickness, WSI and RCE (all $p<0.05$ by ANOVA test), but not basal signal intensity, was observed in the ileum that paralleled the severity of endoscopic lesions. Examples of $M R$ alterations associated with the presence of active inflammation are shown in figs 2 and 3 .

Interobserver agreement in the evaluation of $M R$ changes was very high. The kappa index was $0.96(p<0.001)$ for the presence of oedema, $0.87(p<0.001)$ for ulcers, $0.92(p<0.001)$ for lymph nodes and $0.85(p<0.001)$ for pseudopolyps. The intraclass correlation coefficients were $0.91(p<0.001)$ for wall thickness, $0.73(p<0.001)$ for basal signal intensity and $0.93(p<0.001)$ for contrast signal intensity. 
Table 2 Clinical and biological characteristics of patients at inclusion in the study

\begin{tabular}{|c|c|}
\hline Female, n (\%) & $27(54)$ \\
\hline Age at diagnosis, median (IOR) & $26(20-35)$ \\
\hline Disease duration (years), median (IQR) & $6(2-11)$ \\
\hline \multicolumn{2}{|l|}{ Disease location } \\
\hline Ileal, n (\%) & $11(22)$ \\
\hline Ileocolonic, n (\%) & $24(48)$ \\
\hline Colonic, n (\%) & $15(30)$ \\
\hline Associated upper Gl lesions & $5(10)$ \\
\hline Perianal involvement, $\mathrm{n}(\%)$ & $12(24)$ \\
\hline Harvey-Bradshaw index, median (IQR) & $7(3-11)$ \\
\hline Harvey-Bradshaw index $>4, n(\%)$ & $35(70)$ \\
\hline Previous surgery, $\mathrm{n}(\%)$ & $15(30)$ \\
\hline \multicolumn{2}{|l|}{ Concomitant treatments } \\
\hline 5-ASA, n (\%) & $10(20)$ \\
\hline Steroids, n (\%) & $16(32)$ \\
\hline Immunosuppressants, n (\%) & $24(48)$ \\
\hline Anti-TNF antibodies, n (\%) & $5(10)$ \\
\hline CRP (mg/l), median (IQR) & $19.5(5.0-77.5)$ \\
\hline CDEIS, median (IQR) & $6.68(2.47-13.98)$ \\
\hline CDEIS $<3.5 / 3.5-7 />7, n(\%)$ & $13(26) / 12(24) / 25(50)$ \\
\hline
\end{tabular}

5-ASA, 5-acetylsalicylic acid; CDEIS, Crohn's Disease Endoscopic Index of Severity; CRP, C-reactive protein; $\mathrm{Gl}$, gastrointestinal; IQR, interquartile range; TNF, tumour necrosis factor.

\section{Predictors of disease activity and severity in MR}

To determine which of the MR findings had an independent predictive value for the presence of disease activity at endoscopy, the presence of ulcers at endoscopy or to predict the quantitative CDEIS value for a particular segment, the variable presence of lymph nodes was not introduced in the regression analyses, because this finding is not uniform in all anatomical locations. Since there was a highly significant correlation between contrast signal intensity and RCE, and the latter may be less influenced by technical characteristics of the MR equipment, only RCE was introduced in the regression analyses.

The only MR findings that were independent predictors of the presence of active disease at endoscopy, with either mild or severe lesions, were wall thickness $(p<0.001)$ and RCE $(p=0.013)$. The accuracy of a score based on these variables to predict active disease was high, with an area under the ROC curve of 0.89 (fig 4A), and sensitivity and specificity $>0.8$. We performed an internal validation of the model by bootstrapping, and in 997 replications of random sampling the frequency of occurrence of wall thickness as a predictor was 100\% and that of RCE 54\%.

Logistic regression analysis showed that independent predictors of the presence of ulcers at endoscopy were wall thickness $(p<0.001), \operatorname{RCE}(p=0.03)$ and identification of ulcers at MR $(p=0.003)$. The accuracy of a score based on these variables to predict the presence of ulcerations at endoscopy was high, with an area under the ROC curve of 0.97 (fig 4B), sensitivity of 0.9 and specificity of 0.94 . Bootstrapping analysis showed that the predictors were robust, confirming all the variables from logistic regression, with wall thickness appearing in $91 \%$, presence of ulcer at MR in $73 \%$ and RCE in $57 \%$ of the replications.

Since data distribution for CDEIS of segments was not normal, with $60 \%$ of segments having no significant lesions, a tobit regression analysis was applied. This analysis demonstrated that independent predictors for CDEIS are wall thickness $(p=0.007)$ RCE $(p=0.01)$, presence of oedema
Table 3 Prevalence of qualitative magnetic resonance findings according to endoscopic severity of colonic lesions

\begin{tabular}{lcll}
\hline & Normal mucosa & $\begin{array}{l}\text { Non-ulcerative } \\
\text { lesions* }\end{array}$ & Ulceration \\
\hline No & 130 & 43 & 40 \\
Oedema $\dagger$ & $0 \%$ & $20.9 \%$ & $77.5 \%$ \\
Ulcers $\dagger$ & $0 \%$ & $7.0 \%$ & $65.0 \%$ \\
Pseudopolyps $\dagger$ & $1.5 \%$ & $4.7 \%$ & $27.5 \%$ \\
Enlarged lymph & $0.8 \%$ & $7.0 \%$ & $22.5 \%$ \\
nodes $\dagger$ & & &
\end{tabular}

*Non-ulcerative lesions include erythema, oedema, pseudopolyps and aphtae.

†Comparison of proportions $\mathrm{p}<0.001 \chi^{2}$ test.

$(p=0.02)$ and presence of ulcers at MR $(p=0.003)$. There was a high and significant correlation coefficient between the CDEIS of the segment and the MR index calculated according to the logistic regression analysis coefficients $(r=0.82, p<0.001)$.

From the latter analysis we derived a simplified score (Magnetic Resonance Index of Activity) to quantify disease activity based on MR findings in each segment as follows:

$$
\begin{gathered}
\text { MRIA (segment) }=1.5 \times \text { wall thickness } \\
(\mathrm{mm})+0.02 \times \mathrm{RCE}+5 \times \text { oedema }+10 \times \text { ulceration. }
\end{gathered}
$$

The calculated simplified MRIA index also has a high $(r=0.81)$ and significant $(p<0.001)$ correlation with the CEDEIS of the corresponding segment. The simplified MRIA in fact had a high correlation $(r=0.997)$ with the index calculated directly from the truncated logistic regression. MRIA range was 3.4-35.0; the index has a high accuracy for the detection of disease activity (area under the ROC curve 0.891 , sensitivity 0.81 , specificity 0.89 , using a cut-off point of 7.3) and for the detection of ulcerative lesions (area under the ROC curve 0.978 , sensitivity 0.95 , specificity 0.91 , using a cutoff point of 9.6) in the colon and terminal ileum.

\section{Comparison of MR and other measures of disease activity}

A global MRIA was calculated by adding the values of rectum, sigmoid, descending, transverse and ascending colon and ileum. A significant correlation of the global MRAI index was observed with CDEIS ( $r=0.78, p<0.001$ ) (fig 5), Harvey-Bradshaw index $(r=0.56, p<0.001)$ and CRP $(r=0.53, p<0.001)$. Correlations of CDEIS with the Harvey-Bradshaw index $(r=0.59, p<0.001)$ and CRP $(r=0.42, p<0.01)$ were of similar magnitude to those of the MRAI index.

\section{DISCUSSION}

In this prospective study we provide evidence that the magnitude of quantitative MR changes such as wall thickening, contrast signal intensity and RCE closely parallel the severity of endoscopic lesions; and also the presence of oedema, ulcers, enlarged lymph nodes and pseudopolyps is significantly more prevalent in intestinal segments with more severe endoscopic lesions. This allowed the creation of an MR index of activity composed of the factors with independent predictive value that has a high accuracy for the detection of disease activity and presence of ulceration and is highly correlated with CDEIS.

MR technical advances including ultra-high-field strength body MR, capability of providing fast multiplanar images with excellent soft tissue contrast, requirement for minimally invasive procedures, and lack of radiation exposure allowing repeated assessments over time render $\mathrm{MR}$ a potentially 
Figure 1 Changes in quantitative magnetic resonance (MR) parameters according to endoscopic severity of colonic lesions. Wall thickness (A), postontrast wall signal intensity (C) and relative contrast enhancement (D) were significantly increased in parallel with the severity of endoscopic lesions. Basal signal intensity (B) was not influenced by inflammatory lesions. ${ }^{*} \mathrm{p}<0.01$. w/o, without.
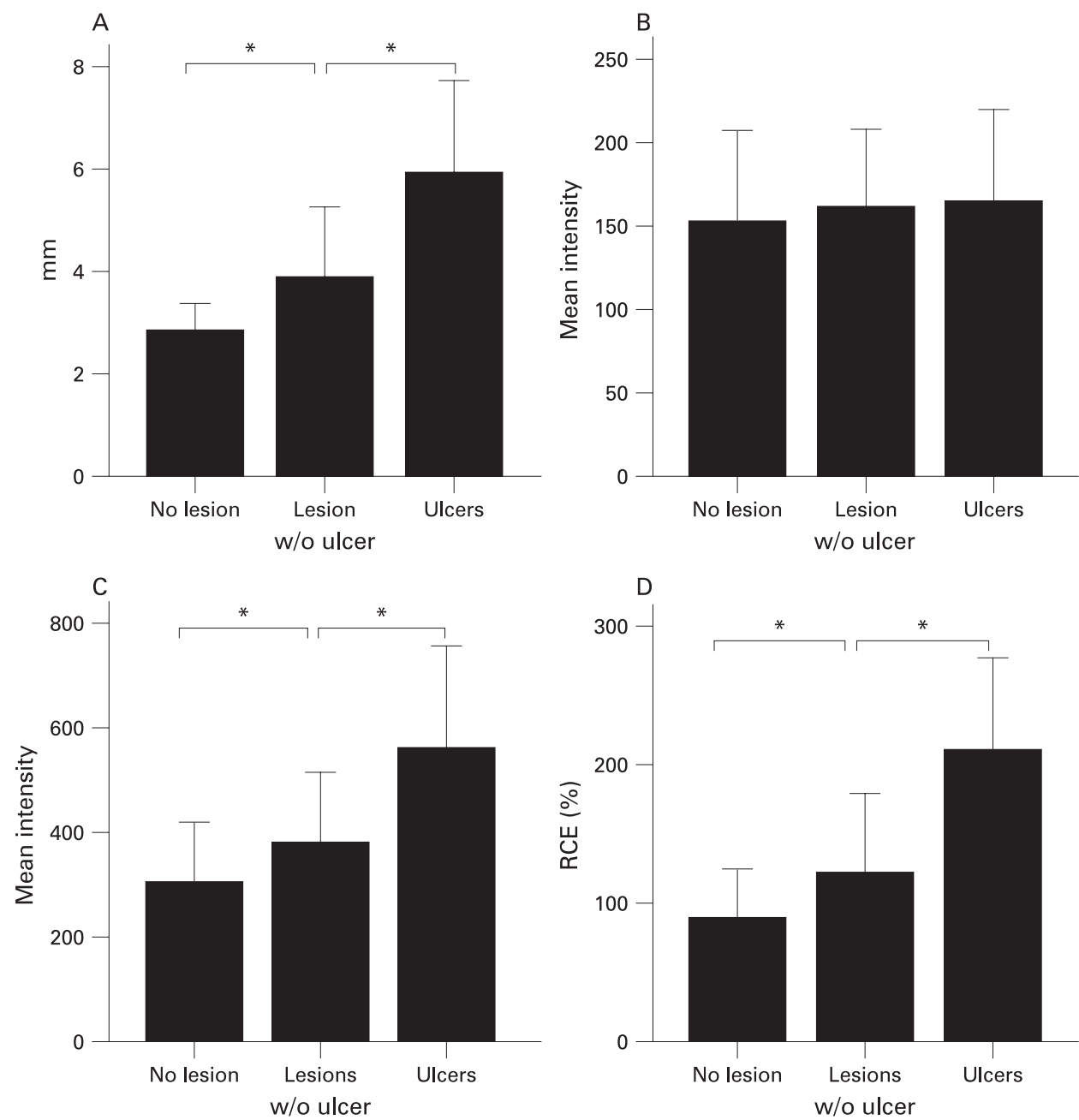

valuable technique for evaluation of patients with CD. ${ }^{14-17}$ Previous studies in patients with $\mathrm{CD}$ have predominantly evaluated the diagnostic potential of $M R$ for small bowel lesions, showing promising results. However, data regarding the diagnostic accuracy of MR for colonic lesions are so far scarce and controversial. Whereas results reported by Narin et $a l^{9}$ and Ajaj et $a t^{5}$ provide an acceptable concordance of findings between MR and endoscopy for the detection of disease activity (sensitivity of $87-89 \%$ and specificity of $85-100 \%$ ), Schreyer et $a l^{8}$ and Dintel et $a l^{6}$ reported only discrete results (sensitivity of
Figure 2 (A) Magnetic resonance axial T2-wedged depicts moderate wall thickening of the ascending colon (arrowheads) that also has a hypersignal relative to the psoas muscle (arrow) indicating the presence of oedema. (B) Coronal T1-VIBE (volumetric interpolated breath-hold examination) after intravenous contrast administration. Marked enhancement of the sigmoid colon and descending colon (arrows) relative to the remaining colon can be seen. Also note the presence of the irregularity of the mucosal surface showing ulcers and inflammatory pseudopolyps.
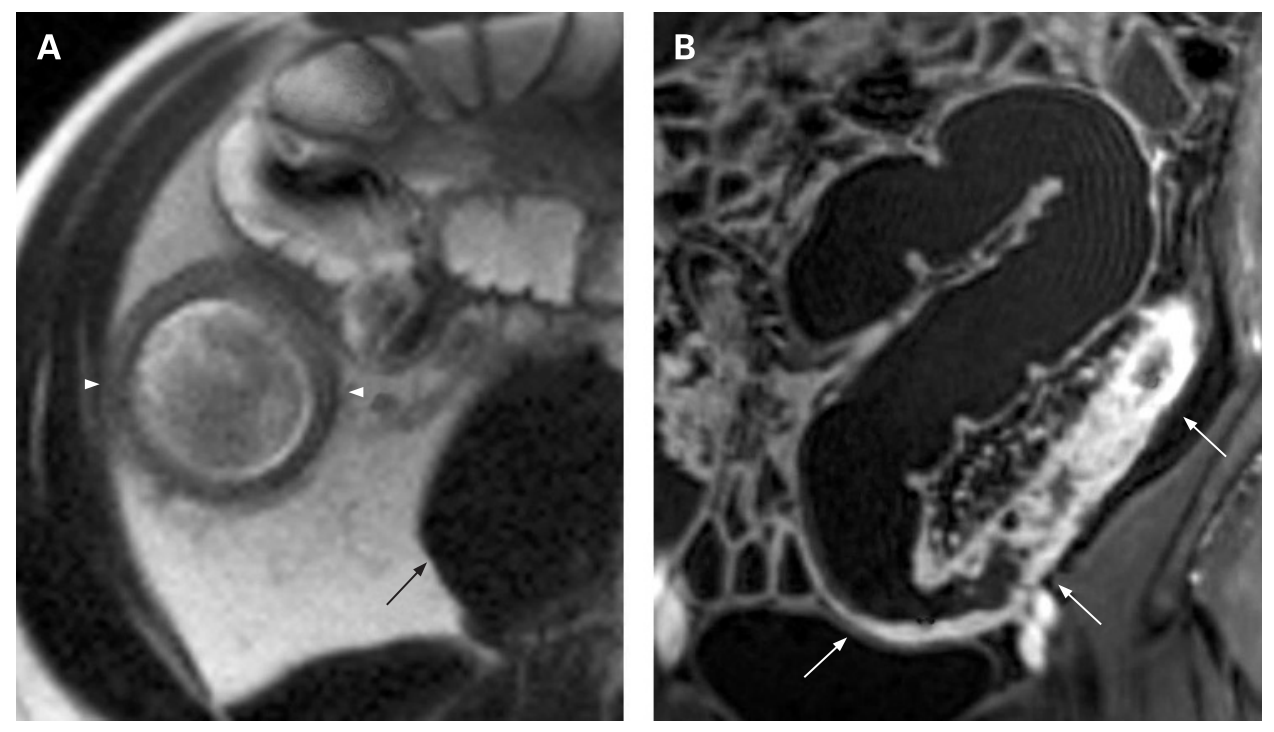
Figure 3 Sagital T1-VIBE (volumetric interpolated breath-hold examination) after intravenous contrast administration of the descending colon and splenic flexure showing discontinuous lesions with segmental thickening of the colon wall and deep ulcers (arrowheads) alternating with a segment without inflammatory changes (arrow) (A); colonoscopy of the same segment shows the presence of deep ulcers in the mucosa (B).
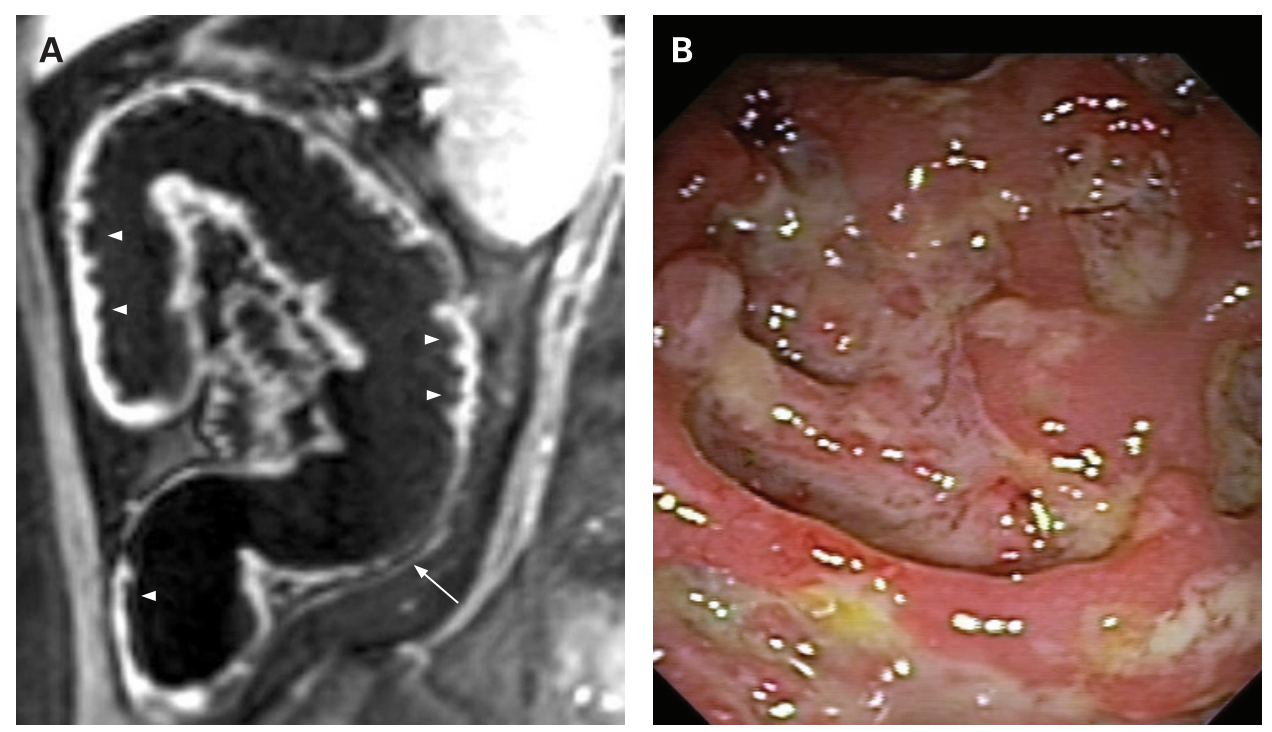

$32-64 \%$ and specificity of $81-100 \%$ ), suggesting that the technique may not be adequate for evaluation of $\mathrm{CD}$ lesions. Discrepancy between these results can be partially explained on the basis of technical aspects. The study of Schreyer et al employed the "bright lumen" MR technique, using a rectal enema of gadolinium that produces a hypersignal on both T1 and T2 sequences and can partially mask the enhancement and mural thickening of the colonic wall. More recently, "dark lumen" MR is increasingly applied, as we did in the current study. Using a water rectal enema produces intraluminal hypointensity on T1 sequences, achieving a high contrast with a bright bowel wall after intravenous contrast administration. In two studies, Dintel et $a l^{6}$ and Langhorst et al evaluated the inflammatory changes in the colon using "dark lumen" MR but without bowel cleansing; this can hamper the identification of inflammatory changes due to difficulties in establishing precise measurements of wall thickness and obscuring the presence of ulcerations. Similar to our study, Narin et al and Ajaj et a ${ }^{5}$ applied "dark lumen" MR with bowel cleansing to evaluate the colon in $\mathrm{CD}$, showing in both cases a high accuracy of MR for the detection of lesions. However, both trials included a relatively small number of patients, and made only a qualitative analysis of MR findings. Considering our results together with previous evidence, we suggest that dark lumen MR with bowel cleansing is probably the current best choice to evaluate inflammatory changes in the colon using this imaging modality. The optimal timing of data acquisition for MR colonography is somewhat controversial. We begin the acquisition $70 \mathrm{~s}$ after the initiation of the bolus, based on previously reported studies. ${ }^{5} 18$ This phase allows assessment of mucosal and mural enhancement as well as visualisation of abdominal viscera. Others have suggested an enterography phase ( $\sim 45 \mathrm{~s}$ after bolus injection) for better detection of mucosal enhancement, but it seems that this provides no contribution to the assessment of CD activity. ${ }^{19}$

An aspect that may have contributed to the high diagnostic accuracy in our study is the use of a 3.0 T MR unit. The images obtained with $3.0 \mathrm{~T}$ have a better signal and higher spatial resolution than those obtained with $1.5 \mathrm{~T}$, contributing to increasing the sensitivity for lesion detection. ${ }^{17}{ }^{20}$ Some artefacts are associated with the use of a 3.0 T MR unit, in particular magnetic susceptibility artefacts that appear on T2 sequences in the presence of paramagnetic objects (eg, surgical suture in ileocolic anastomosis). Nevertheless, these artefacts do not usually represent a limitation for evaluation of potential inflammatory changes because the extension of the inflammatory tissue is larger than the artefact. Also, an additional
Figure 4 Receiver operating characteristic (ROC) curves, of a magnetic resonance-based index to predict presence of active disease $(A)$ and presence of ulcerative lesions $(B)$ in Crohn's disease.
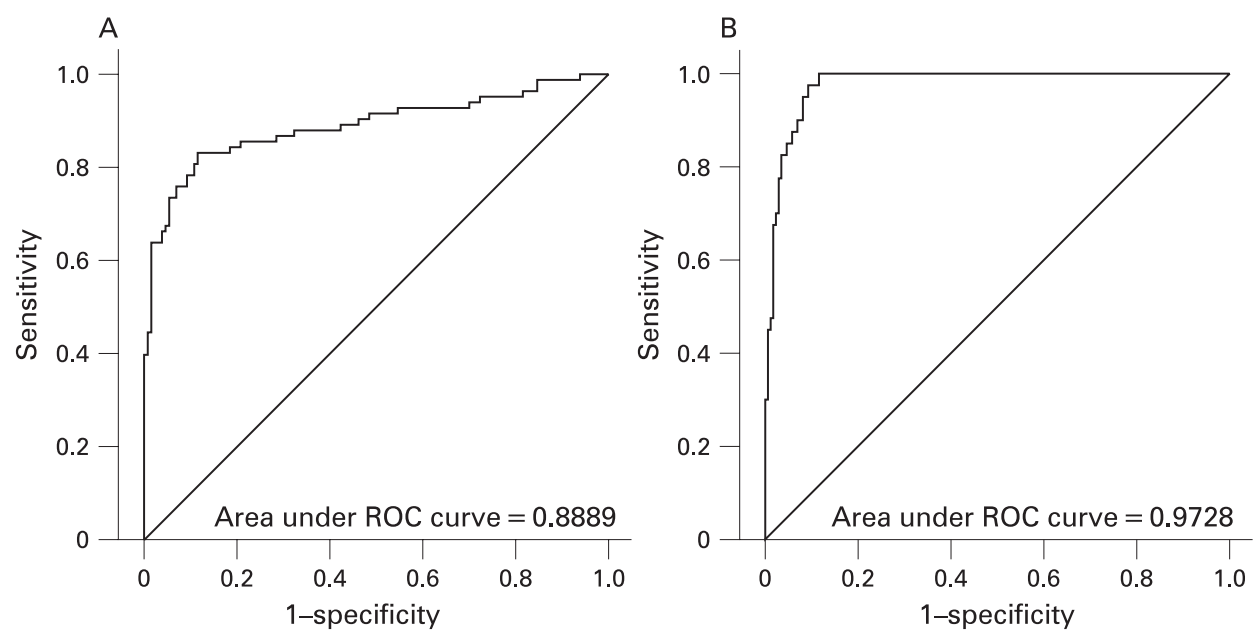


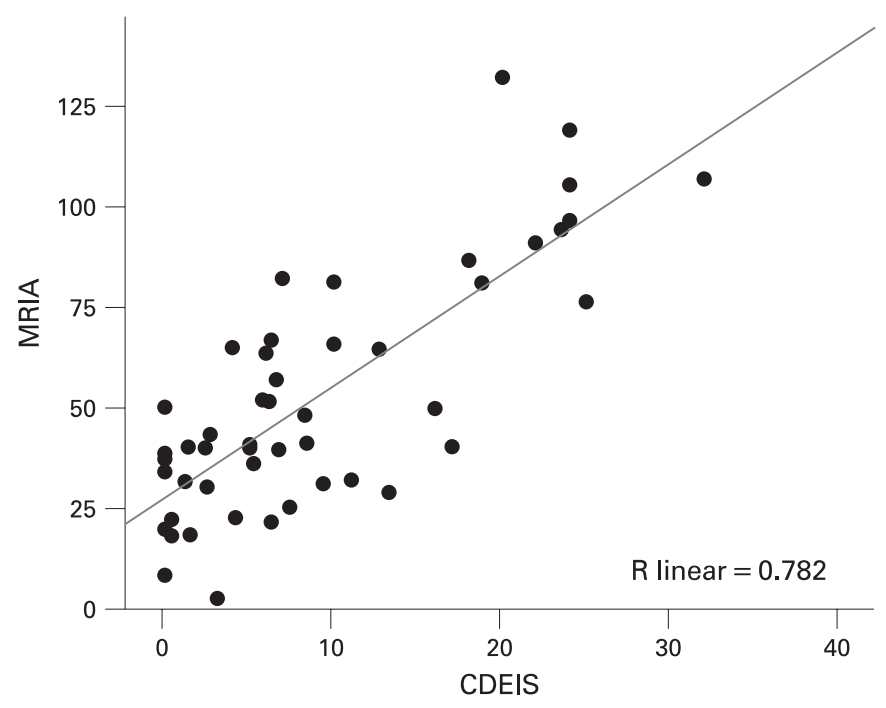

Figure 5 Correlation between the Crohn's Disease Endoscopic Index of Severity (CDEIS) and the global Magnetic Resonance Index of Activity (MRIA).

measure to reduce susceptibility artefacts is the use of fast turbo spin-echo T2 sequences (eg, HASTE). ${ }^{21}$

Both MR and CT techniques emerge as excellent techniques to assess inflammatory activity and detection of extraintestinal complications in CD. ${ }^{21422-24}$ CT provides a better spatial resolution than $M R$, has greater availability and is less time consuming. However, MR provides better soft tissue contrast and uses non-ionising radiation. It has been estimated that CT examinations account for most of the irradiation exposure in patients with IBD. ${ }^{35}$ The long-term cumulative effect of this radiation in combination with co-exposure to potentially synergistic agents such as methotrexate may increase the risk of neoplasia in these patients. ${ }^{25}$ Finally, as for MR, studies on the accuracy of CT in the evaluation of CD focused on the detection of lesions in the small bowel. The only prospective study evaluating the value of CT for the characterisation of inflammatory lesions in the colon suggests a limited sensitivity of CT, although only the mucosal alterations, and not wall thickness and contrast enhancement, were considered. ${ }^{26}$

Doppler ultrasonography represents another non-ionising imaging technique which may provide information about disease activity. ${ }^{27}{ }^{28}$ However, the difficulty of visualisation of deep bowel segments and high interobserver variability represent significant drawbacks. Finally, 99mTc hexamethyl-propylamine-oxim leucocyte scintigraphy is a non-invasive technique, but radiation exposure and limited sensitivity, especially in patients receiving steroid treatment, are leading to a reduced used of this technique. ${ }^{29}$

MR examination of the small bowel requires oral luminal contrast to achieve adequate distension. MR enteroclysis allows better small bowel distention than MR follow-through. However, nasojejunal tube placement entails radiation exposure and produces discomfort, and consequently movement artefacts are more frequent. The only study comparing both modalities concluded that bowel distension was inferior in MR followthrough, but diagnostic accuracy was similar in both methods. ${ }^{30}$ In our study, oral ingestion of the luminal contrast provided adequate distension of the distal ileum, the segment we compared with endoscopy. MR enteroclysis might be necessary in selected cases in which upper CD lesions are suspected and adequate distension is not achieved with oral administration of the luminal contrast.

Because the location of CD lesions in the intestine has a characteristic skip pattern, in which segments with severe ulcerative lesions can be adjacent to others with normal mucosa, we evaluated the MR findings associated with lesions of different endoscopic severity in a segment by segment analysis as in previous studies ${ }^{4}{ }^{24}$ and also as a global MR index of activity. MR changes found to be associated with disease activity and severity in the current study include oedema, presence of ulcers, wall thickening and RCE. In our study, these four parameters were independent predictors of the presence and severity of disease activity. This finding is in keeping with previous studies focused mainly on the small intestine. ${ }^{5} 143132$ Mural oedema is a characteristic feature of active inflammation of the bowel wall, and can be detected as an increase of signal on T2-wedged sequences. In severe involvement, odema can extend to the mesentery. Mural thickening is well correlated with the presence of activity and with severity. Using a threedimensional VIBE sequence, wall thickening can be evaluated at any plane of space. Based on both bowel wall thickening and RCE, inflammatory changes can be detected with great accuracy and played a dominant role in the detection of bowel inflammation.

Other parameters previously related to inflammatory changes include enlarged mesenteric lymph nodes and loss of haustra folds. ${ }^{45}$ We did not include haustra length in our analysis because of its variability between the proximal and distal colon. Also, long-standing $\mathrm{CD}$ can induce shortening of colonic haustra secondary to fibrosis in the submucosa. ${ }^{33}$ The presence of enlarged lymph nodes has great variability depending on the anatomic location and patient age (more frequent in the young population). ${ }^{34}$ Additionally, prevalence of enlarged lymph nodes in our series was low.

Demonstration of the high accuracy of MR in detecting the presence of disease activity and differentiating the presence or absence of ulcers is of considerable clinical relevance given the prognostic implications of the presence or persistence of ulcerative lesions in terms of disease course and surgical requirements. ${ }^{35}$ The MRIA we propose in the current study may be useful to categorise disease severity in a given patient, and to monitor responses to therapeutic interventions both in the clinical setting and in therapeutic trials.

One of the limitations of the current study is the lack of validation of the results in an independent cohort. While these results are not available, we provide an internal validation of the results using bootstrapping. The variables that were independent predictors of the presence of disease activity or the presence of ulcerations were validated by this approach. Also, the magnitude of changes in MRIA in response to treatment and their correlation with changes in the CDEIS need to be characterised in future studies. Furthermore, development of an effective tagging method will probably improve the patient acceptance of MR since one of the main drawbacks of the technique is the need for bowel cleansing.

Overall, the results provided in the current study lead to the suggestion that $\mathrm{MR}$ may be considered as an alternative to endoscopy in cases with an established diagnosis of ileocolonic $\mathrm{CD}$ in which biopsy samples are not necessary. MR has significant advantages over colonoscopy, including the ability provide a complete assessment of the ileocolonic region in all patients, as compared with $72 \%$ of complete ileocolonoscopies in our series, a proportion very similar to previous observations. As performed in our study, administration of oral contrast and 
an enema for MR may produce slight discomfort in some patients, but anaesthesia is not required as for ileocolonoscopy. The possibility of identifying inflammatory lesions in all locations of the gastrointestinal tract, and also extraluminal complications, in just one examination represents a clear advantage of MR over colonoscopy for evaluation of CD.

Acknowledgements: This work has been supported by grant PI07/90253 from the Ministerio de Sanidad, Spain.

Competing interests: None.

Ethics approval: The ethics committee of the Hospital Clínic of Barcelona approved the project

\section{REFERENCES}

1. Mary JY, Modigliani R. Development and validation of an endoscopic index of the severity for Crohn's disease: a prospective multicentre study. Groupe d'Etudes Therapeutiques des Affections Inflammatoires du Tube Digestif (GETAID). Gut 1989;30:983-9.

2. Bodily KD, Fletcher JG, Solem CA, et al. Crohn disease: mural attenuation and thickness at contrast-enhanced CT enterography-correlation with endoscopic and histologic findings of inflammation. Radiology 2006;238:505-16.

3. Brenner DJ, Hall EJ. Computed tomography - an increasing source of radiation exposure. N Engl J Med 2007;357:2277-84.

4. Bru C, Sans M, Defelitto MM, et al. Hydrocolonic sonography for evaluating inflammatory bowel disease. AJR Am J Roentgenol 2001;177:99-105.

5. Ajaj WM, Lauenstein TC, Pelster G, et al. Magnetic resonance colonography for the detection of inflammatory diseases of the large bowel: quantifying the inflammatory activity. Gut 2005;54:257-63.

6. Dinter DJ, Chakraborty A, Brade J, et al. Endoscopy and magnetic resonance imaging in patients with Crohn's disease: a retrospective single-centre comparative study. Scand J Gastroenterol 2008;43:207-16.

7. Langhorst J, Kuhle CA, Ajaj W, et al. MR colonography without bowel purgation for the assessment of inflammatory bowel diseases: diagnostic accuracy and patient acceptance. Inflamm Bowel Dis 2007;13:1001-8.

8. Schreyer AG, Rath HC, Kikinis R, et al. Comparison of magnetic resonance imaging colonography with conventional colonoscopy for the assessment of intestinal inflammation in patients with inflammatory bowel disease: a feasibility study. Gut 2005;54:250-6.

9. Narin B, Ajaj W, Gohde S, et al. Combined small and large bowel MR imaging in patients with Crohn's disease: a feasibility study. Eur Radiol 2004;14:1535-42.

10. Harvey RF, Bradshaw JM. A simple index of Crohn's disease activity. Lancet 1980;1:514.

11. Semelka RC, Shoenut JP, Silverman R, et al. Bowel disease: prospective comparison of CT and 1.5-T pre- and postcontrast MR imaging with T1-weighted fatsuppressed and breath-hold FLASH sequences. J Magn Reson Imaging 1991;1:625-32.

12. Fleiss J. Statistical methods for rates and proportions. New York: John Wiley and Sons, 1981.

13. Shrout $\mathbf{P}$, Fleiss JL. Intraclass correlations: uses in assessing rater reliability. Psychol Bull 1979;86:420-8.

14. Gourtsoyiannis NC, Grammatikakis J, Papamastorakis G, et al. Imaging of small intestinal Crohn's disease: comparison between MR enteroclysis and conventional enteroclysis. Eur Radiol 2006;16:1915-25.
15. Koh DM, Miao Y, Chinn RJ, et al. MR imaging evaluation of the activity of Crohn's disease. AJR Am J Roentgenol 2001;177:1325-32.

16. Umschaden HW, Szolar D, Gasser J, et al. Small-bowel disease: comparison of MR enteroclysis images with conventional enteroclysis and surgical findings. Radiology 2000;215:717-25.

17. Choi JY, Kim MJ, Chung YE, et al. Abdominal applications of 3.0-T MR imaging: comparative review versus a 1.5-T system. Radiographics 2008;28:e30.

18. Maccioni F, Bruni A, Viscido A, et al. MR imaging in patients with Crohn disease value of T2- versus T1-weighted gadolinium-enhanced MR sequences with use of an oral superparamagnetic contrast agent. Radiology 2006;238:517-30.

19. Wold PB, Fletcher JG, Johnson CD, et al. Assessment of small bowel Crohn disease: noninvasive peroral CT enterography compared with other imaging methods and endoscopy-feasibility study. Radiology 2003;229:275-81.

20. Akisik FM, Sandrasegaran K, Aisen AM, et al. Abdominal MR imaging at $3.0 \mathrm{~T}$. Radiographics 2007;27:1433-44; discussion 62-4.

21. Barth MM, Smith MP, Pedrosa I, et al. Body MR imaging at 3.0 T: understanding the opportunities and challenges. Radiographics 2007;27:1445-62; discussion 62-4.

22. Paulsen SR, Huprich JE, Fletcher JG, et al. CT enterography as a diagnostic tool in evaluating small bowel disorders: review of clinical experience with over 700 cases. Radiographics 2006;26:641-57; discussion 57-62.

23. Colombel JF, Solem CA, Sandborn WJ, et al. Quantitative measurement and visual assessment of ileal Crohn's disease activity by computed tomography enterography: correlation with endoscopic severity and C reactive protein. Gut 2006;55:1561-7.

24. Ajaj W, Lauenstein TC, Pelster G, et al. MR colonography in patients with incomplete conventional colonoscopy. Radiology 2005;234:452-9.

25. Jaffe TA, Gaca AM, Delaney S, et al. Radiation doses from small-bowel followthrough and abdominopelvic MDCT in Crohn's disease. AJR Am J Roentgenol 2007; 189:1015-22.

26. Ota $\mathbf{Y}$, Matsui T, Ono $\mathrm{H}$, et al. Value of virtual computed tomographic colonography for Crohn's colitis: comparison with endoscopy and barium enema. Abdom Imaging 2003;28:778-83.

27. Martinez MJ, Ripolles T, Paredes JM, et al. Assessment of the extension and the inflammatory activity in Crohn's disease: comparison of ultrasound and MRI. Abdom Imaging 2008.

28. Maconi G, Radice E, Greco S, et al. Bowel ultrasound in Crohn's disease. Best Pract Res Clin Gastroenterol 2006;20:93-112.

29. Sans M, Fuster D, Llach J, et al. Optimization of technetium-99m-HMPAO leukocyte scintigraphy in evaluation of active inflammatory bowel disease. Dig Dis Sci 2000;45:1828-35.

30. Negaard A, Paulsen V, Sandvik L, et al. A prospective randomized comparison between two MRI studies of the small bowel in Crohn's disease, the oral contrast method and MR enteroclysis. Eur Radiol 2007;17:2294-301.

31. Bernstein CN, Greenberg H, Boult I, et al. A prospective comparison study of MRI versus small bowel follow-through in recurrent Crohn's disease. Am J Gastroenterol 2005; 100:2493-502.

32. Albert JG, Martiny F, Krummenerl A, et al. Diagnosis of small bowel Crohn's disease: a prospective comparison of capsule endoscopy with magnetic resonance imaging and fluoroscopic enteroclysis. Gut 2005;54:1721-7.

33. Gore RM, Balthazar EJ, Ghahremani GG, et al. CT features of ulcerative colitis and Crohn's disease. AJR Am J Roentgenol 1996;167:3-15.

34. Maconi G, Di Sabatino A, Ardizzone S, et al. Prevalence and clinical significance of sonographic detection of enlarged regional lymph nodes in Crohn's disease. Scand J Gastroenterol 2005;40:1328-33.

35. Allez M, Lemann M, Bonnet J, et al. Long term outcome of patients with active Crohn's disease exhibiting extensive and deep ulcerations at colonoscopy. Am J Gastroenterol 2002;97:947-53. 


\section{GUT}

\section{Magnetic resonance for assessment of disease activity and severity in ileocolonic Crohn's disease}

J Rimola, S Rodriguez, O García-Bosch, et al.

Gut 2009 58: 1113-1120 originally published online January 9, 2009 doi: 10.1136/gut.2008.167957

Updated information and services can be found at:

http://gut.bmj.com/content/58/8/1113.full.html

These include:

References This article cites 30 articles, 14 of which can be accessed free at: http://gut.bmj.com/content/58/8/1113.full.html\#ref-list-1

Article cited in:

http://gut.bmj.com/content/58/8/1113.full.html\#related-urls

Email alerting

Receive free email alerts when new articles cite this article. Sign up in service the box at the top right corner of the online article.

Notes

To request permissions go to:

http://group.bmj.com/group/rights-licensing/permissions

To order reprints go to:

http://journals.bmj.com/cgi/reprintform

To subscribe to BMJ go to:

http://group.bmj.com/subscribe/ 